\title{
Cotton Leaf Image Segmentation using Modified Factorization-Based Active Contour Method
}

\author{
Bhagya M Patil ${ }^{1}$ \\ School of Computer Science \\ REVA University, Bengaluru, India
}

\author{
Dr. Basavaraj Amarapur² \\ Department of Electrical and Electronics Engineering \\ PDA College of Engineering, Kalaburgi, India
}

\begin{abstract}
Cotton plant is one of the most widely cultivated crop across worldwide. The leaf is one of the important parts which help in the food production. There are different cotton leaf diseases like Alternaria spot, foliar, bacterial blight, etc. which affects the agricultural yield. In order to detect the diseases, leaf region extraction becomes a significant task and to achieve this we use image processing techniques. Henceforth in this paper, a novel method used to extract the leaf region from a complex background. The proposed method is used for leaf extraction from complex background. The algorithm used in this method is modified factorization based active contour (MFACM) which helps in getting better output images. The database images used for research are acquired from the field using a digital camera. The proposed work is compared with existing active contour algorithms like Gradient Vector Flow (GVF), Adaptive Diffusion Flow (ADF), and Vector Flow Convolution (VFC). From the experiment, it can be observed that the proposed method is better than the other active contour methods in terms of computation time and the number of iterations. In addition to that segmented result is analyzed using specificity, sensitivity, precision which showed that our proposed method is better than the other methods.
\end{abstract}

Keywords-Cotton leaf; active contour; Gradient Vector Flow (GVF); Adaptive Diffusion Flow (ADF); Vector Flow Convolution (VFC); Modified factorization based active contour (MFACM)

\section{INTRODUCTION}

Plants play an important source of food for human beings. If plants get affected than yield will also get affected. Therefore, many researchers are into this field of plant phenotyping [7] and there has been extensive research in this area. Plant phenotyping is a research area where the quantitative measurements of the structural and functional properties are performed. The experiment is carried out using some of the computer vision methods like analyzing the image through software. Because of this there has been research in this field for plant disease classification, leaf counting, observing the development and growth of the plant. In literature, there are many researchers who did lot of work for segmenting the leaf images [15,25,26,27] for example leaf segmentation without background and with complex background, automatic identification of plant species. In some cases, segmentation is performed using edge based and local based method also is used [16].

Kiruba raji [2], proposed a method for the herbal leaves segmentation from the complex background. In this chan-vese method is used for the segmentation of leaf or leaves from background when compared to other techniques like $\mathrm{k}$ means clustering, local adaptive mean color, without affecting the color, textures. J Praveen kumar [3], introduced the new edge enhancement technique and graph-based method to extract the leaf region. Later it involves counting the number of leaves using circular Hough transform. Manual Grand et al. [4], a review of comparative study of 13 different segmentation methods. The Guided active contour approach was one among them and it has good segmentation results. Jones De Kylder [6], proposed a new active contour framework for segmentation. In this, the author proposed that whenever at the edge of the object contour is placed at that moment it will maximize probability. The internal and external probability distribution functions are learned from a ground truth training set. So, using this segmentation is performed and it gave an outstanding result. Shivalika Sharma [7], presented the review of various methods available for leaf segmentation. Xiaodong Tang [10], introduced leaf extraction from a complicated background using marker-controlled watershed algorithm for image of the individual channel gradient image of Hue, Saturation and Intensity separately. The applying of the watershed algorithm leads to the segmentation of the leaf region and author used solidity measures, to know how the performance of the method is.

Though there are various techniques used for the leaf segmentation [1][12][17][28] but with complex background very less research is carried out. Taking this into consideration, we proposed a leaf segmentation from complex background using modified factorization based active contour for texture segmentation. The structure of the paper is as follows: Section II is Literature survey: discussed about previous work done; Section III is Methodology: explains regarding proposed method; Section IV is Results and discussion: the comparative study results; and Section V presents Conclusion.

\section{LITERATURE SURVEY}

In this, we will be focusing on the previous work done by researchers in active contours, or snakes. Active contour are curves which are generated by the computer. The curve will move towards the edges of an object based on the image energies. There are various applications of active contours like in the medical field like to outline tumor in the brain or it may be for the number recognition etc. But it has some disadvantages too like if the object boundary is too far than its difficult of the curve to evolve towards the object boundaries. 
Active contours which there are two kinds, namely parametric and geometric.

1) The parametric deformable model -curves which are moved under the influence of internal and external forces $[19,22]$.

2) Geometric deformable model - level set function is used to represent curves and surfaces implicitly in higherdimensional scalar function [18,20,21].

There are various applications of these models in wide range of medical field [24]. But apart from that its contribution is in leaf segmentation also. And in this paper, both methods are taken into consideration for experiment.

\section{A. Gradient Vector Flow}

$\mathrm{Xu}$ and Prince [5], introduced a gradient vector flow method to overcome the problems of traditional snake. The traditional snake had a problem with the convergence towards the concave region and also with the limited capture range. So, in this author introduced a new external force which helps the snake to move towards the concave regions.

The equation for the traditional snake is given by

$E_{\text {int }}=\int_{0}^{1} E_{\text {snake }}(v(s)) d s$

$=\int_{0}^{1} E_{\text {int }}(v(s))+E_{\text {ext }}(v(s))$

Where $E_{\text {int }}$ and $E_{\text {ext }}$ are the internal and external energies. Eext is calculated from the image. In this author proposed a new external force which is given by.

$E_{\text {ext }}(v(s))=-\left|\nabla\left[G_{\sigma}(x, y) * I(x, y)\right]\right|^{2}$

Where $\operatorname{Go}(\mathrm{x}, \mathrm{y})$ is a two dimensional Gaussian function with standard deviation $\sigma$ and $\nabla$ is the gradient operator. This energy helps in moving the snake towards the edges.

It will give better performance with respect to deep concavities of an image.

\section{B. Adaptive Diffusion Flow}

Yuwei Wu [8], proposed a method of adaptive diffusion flow which is the modified version of gradient vector flow. Gradient vector flow has advantages with respect to reaching deep concavities but it had a problem with the leakage of weak edges and narrow concavity. So, to improve the author replaced the smoothness energy term of GVF to harmonic hyper surface minimal functional and to achieve the contour to the deep and narrow concavities the infinite laplacian functional is incorporated.

Following are the equations for the adaptive diffusion flow which helps in overcoming the problems of GVF.

Case i): To preserve the weak edges following is the harmonic hypersurface functional defined as.

$E(v)=\iint \frac{1}{p|\nabla f|} \cdot\left(\sqrt{1+\left|G_{\sigma} \otimes \nabla v\right|^{2}}\right)^{p|\nabla f|}$

Where $\mathrm{f}$ is the edge of the image $\mathrm{I}, \mathrm{p}(\cdot)$ is a decreasing monotonously function which ranges from 1 to 2 . And $p(\nabla f)=1+\frac{1}{1+\left|\nabla G_{\sigma} \otimes \mathrm{f}(\mathrm{x})\right|}$ such that when $\left|\nabla G_{\sigma} \otimes \mathrm{f}(\mathrm{x})\right| \rightarrow 0, \mathrm{p}(\cdot)$ $\rightarrow 2$, this function behaves like isotropic diffusion within homogenous regions. When $\left|\nabla G_{\sigma} \otimes \mathrm{f}(\mathrm{x})\right| \rightarrow \infty, \mathrm{p}(\cdot) \rightarrow 1$ behaves like total variation model. This basically helps in preserving the weak edges of an image.

Case ii): The laplacian energy functional helps in contour convergence of narrow and deep concavities of an image. Following is the laplacian energy functional.

$E_{\infty} v=\int\left|\nabla G_{\sigma} \otimes \nabla \mathrm{v}\right|_{L^{\infty}(\Omega)} d \Omega$

So, using both the cases the adaptive diffusion method is given by.

$$
\begin{aligned}
& E(u)=\iint\left[g \cdot\left(-m \cdot\left|G_{\sigma} \otimes \nabla u\right|_{L^{\infty}}\right]+(1-m) \cdot \frac{1}{p(|\nabla f|)} .\right. \\
& \left.\left.\left(\sqrt{1+\left|G_{\sigma} \otimes \nabla u\right|^{2}}\right)^{p|\nabla f|}\right)+h \cdot\left(\left|u-f_{x}\right|^{2}\right)\right] d \Omega
\end{aligned}
$$

Where $\mathrm{g}, \mathrm{h}, \mathrm{m}$ are the weighting functions.

\section{Vector Flow Convolution}

Bing Li [9], introduced new external force called as vector field convolution (VFC). Here the external force vector field kernel $\mathrm{k}(\mathrm{x}, \mathrm{y})$ in which all the vectors point to the kernel origin.

$\mathrm{k}(\mathrm{x}, \mathrm{y})=\mathrm{m}(\mathrm{x}, \mathrm{y}) \mathrm{n}(\mathrm{x}, \mathrm{y})$

The convolution of kernel $k(x, y)$ with edge map $f(x, y)$ which is generated from the image $\mathrm{I}(\mathrm{x}, \mathrm{y})$ which is considered as VFC external force.

$\mathrm{fvfc}=\mathrm{f}(\mathrm{x}, \mathrm{y}) * \mathrm{k}(\mathrm{x}, \mathrm{y})$

Here edges are more prominent compare to homogeneous regions. The VFC will result in edge map which doesn't depend on the origin of the vector field kernel but it depends on the magnitude of the vector field kernel $\mathrm{m}(\mathrm{x}, \mathrm{y})$. Author proposed a two magnitude functions given by.

$m_{1}(x, y)=(r+\epsilon)^{-\gamma}$

$m_{2}(x, y)=\exp \left(\frac{-r^{2}}{\zeta^{2}}\right)$

Where $\gamma$ and $\zeta$ are positive parameters to control the decrease of vector field, $\varepsilon$ is a positive constant to prevent division by zero at the origin.m $1(\mathrm{x}, \mathrm{y})$ which influence the FOI as increases as $\gamma$ decreases. $\mathrm{m} 2(\mathrm{x}, \mathrm{y})$ is a Gaussian shape function and $\zeta$ represents the standard deviation.

\section{Level Set Method}

Stanley Osher [29], introduced the level set method formulation for the evolution of the curve. The curve is represented implicitly as a level set of a scalar function referred as level set function which is defined as the set of points that have same function value. Fig. 1 shows the curve at zero level set of a function, $\Phi(\mathrm{x})=0$.

\section{E. Drawbacks}

One of the drawbacks, reinitialization of the curve won't be possible whenever the level set function is far away from a signed distance. Practically whenever the time step is not selected small enough at that time evolving level set function 
can depart greatly from its value as signed distance in a small number of iteration steps.

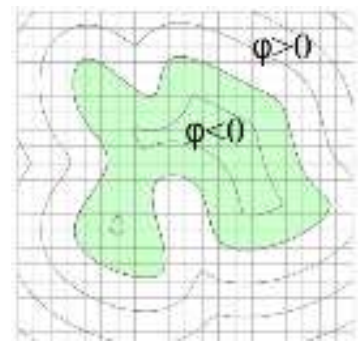

Fig. 1. Curve at Zero Level Set.

To maintain curve evolution stability and guaranteeing required results re-initialization has been broadly used as a numerical remedy. Later, various authors came with different methods which helped in overcoming the cost of reinitialization of curve during evolution.

Chumming Li [23], came with level set evolution without reinitialization cost which can be achieved by forcing the level set function to be close to signed distance function. The internal energy is used for deviation of the level set function from a signed distance function and external energy based on the desired feature like object boundary are used for the motion of zero level set curve.

\section{Methodology}

\section{A. Leaf Database}

There are nearly 500 images for database. The images are captured under suitable conditions using Nikon digital camera of size $6000 \times 4000$.The images are resized nearly to $256 \times 256$ so that processing of images is faster. Fig. 2 shows the sample leaf images.

There are two different types of deformable models parametric and geometric. ADF, VFC, GVF are the methods for leaf segmentation and modified factorization based active contour.

\section{B. Proposed Method}

The proposed method is based on factorization-based active contour for texture segmentation [11][14] with modification. In geometric deformable the distance regularized active contour is used for the implementation [13]. The method is modified version of a factorization based active contour model for texture segmentation [11]. So, the process of extracting the leaf region shown in Fig. 3.

\section{Modified Factorization based Active Contour Method (MFACM)}

The images which are captured will undergo into preprocessing stage of flattening the field correction and using Gaussian pyramid.
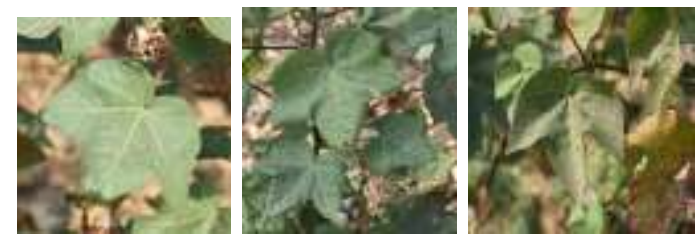

Fig. 2. Sample Leaf Images.

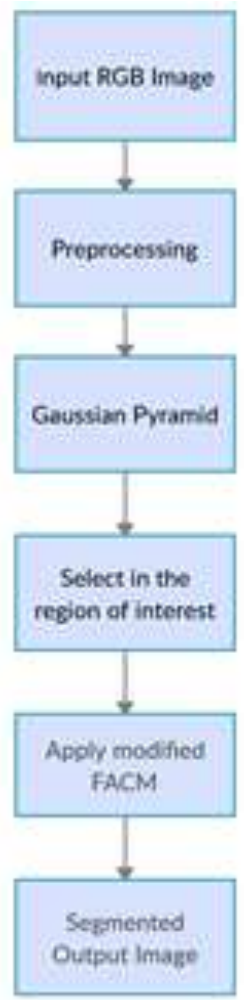

Fig. 3. Process of Extracting the Leaf Region.

The Gaussian pyramid is a technique in image processing that breaks down an image into successively smaller groups of pixels, in repeated steps, for the purpose of blurring it. This is performed so that it is easier to detect the edges of an object.

The proposed method uses level set method introduced by chumming li [13], distance regularization is given by.

$E_{\text {regularization }}(\phi)=\int_{\Omega} \frac{1}{2}(|\nabla \phi(x)-1|)^{2} d x$

Where $\nabla \phi$ denote the derivative of the level set method. The energy functional proposed by the author is defined as.

$E(\phi, R)=\mu E_{\text {data }}(\phi, R)+v E_{\text {regularization }}(\phi)$

where $\mu$ and $\mathrm{v}$ are two positive constant which helps in balancing the total energy with respect to the corresponding terms in the equation. The equation is based on gradient based method.

The evolution process is defined as follows

$\frac{\partial \phi}{\partial t}=-\frac{\partial E(\phi, R)}{\partial \phi}=-\delta_{\epsilon}(\phi) \mu\left(w_{o}-w_{b}\right)+v\left(\nabla^{2} \phi-\operatorname{div}\left(\frac{\nabla \phi}{|\nabla \phi|}\right)\right)$

Where $\mathrm{w}_{\mathrm{o}}$ and $\mathrm{w}_{\mathrm{b}}$ are $\mathrm{N}$ dimension vectors.

Apart from level set method the proposed uses factorization-based texture segmentation introduced by Yaun et al. In this, spectral histograms are considered as texture features and a MxN matrix is calculated representing the feature matrix consisting of local window centered at each pixel. The matrix is denoted by $\mathrm{Y}$ which is given as. 
$\mathrm{Y}=\mathrm{R} \beta+\varepsilon$

Where $\mathrm{R}$ is an MxL matrix gives you the columns which represent the features for the region to be segmented. $\beta$ is the columns representing the weight vectors for every region and is of $\mathrm{LxN}$ matrix, $\varepsilon$ is the additive noise.

The data energy can be represented as

$E_{\text {data }}(\phi, R)=$

$-\int_{\Omega}\left[H_{\epsilon}(\phi) w_{o}(x, R)+\left(1-H_{\epsilon}(\phi) w_{b}(x, R)\right] d x\right.$

From the above equation 9 it can be observed that when the curve reaches the object boundary it data term will have minimum value at that point.

In this proposed method, we have included Gaussian pyramid along with factorization based active contour method.

\section{Algorithm}

Step 1: Read the original image.

Step 2: RGB to Gray scale image.

Step 3: Histogram of RGB is matched with its gray scale image.

Step 4: Flat field correction with sigma $=1$.

Step 5: Gaussian low pass filter with hzise $=[55]$ and sigma $=$ 10.

Step 6: Subtracting ' $q$ ' image from blurred image.

Step 7: Reducing the flat filed corrected image using Gaussian pyramid.

Step 8: Expanding the reduced image IR.

Step 9: Fuse expanded image 'IR' and flat flied correction image ' $\mathrm{O}$ '.

Step 10: Fuse Q and subtracted image q.

Step 11: Flat field correction is applied to Q.

Step 12: Image intensity values are adjusted with gamma = 0.01 .

Step 13: Flat field correction is applied with sigma $=5$.

Step 14: Required region should be selected from the original image.

Step 15: Segmentation is performed using modified factorization active contour method.

Step 16: Segmentation output.

\section{RESUltS AND QuANTITATIVE COMPARISONS}

The images with complex background are captured from a various agriculture field of cotton plant leaf images. The images are captured from a digital camera of size $6000 \times 4000$.

The images were preprocessed using flat field correction and used Gaussian pyramid for the better visibility of the edges of the images. Later the processed image will be used for factorization based active contour. The results obtained using modified factorization based active contour will give better results when compare to other results.

The proposed method and parametric deformable model methods Gradient Vector Flow (GVF), Adaptive Diffusion Flow (ADF), Vector Field Convolution (VFC) are experimented with dataset created by Nikon digital camera of image resolution 6000x4000. In this paper, Modified Factorization based Active Contour Method (MFACM) is compared with the existing parametric deformable models.

Fig. 4 and 5 shows the results obtained after applying the modified FACM, GVF, ADF and VFC methods along with ground truth image. The performance of the methods are analyzed using time computation and segmentation measures like precision, accuracy, sensitivity, specificity. Table I gives the details regarding the time computation obtained from the various methods and we can observe that our proposed method takes less time when compare to other.

Fig. 6 shows the graph of time taken versus the number of iterations. Table II displays the output of different segmentation performance measures using ground truth images. From the table it can be seen that accuracy, precision, sensitivity, etc. are more compare to methods of parametric deformable models and it's been represented in the graph shown in Fig. 7.

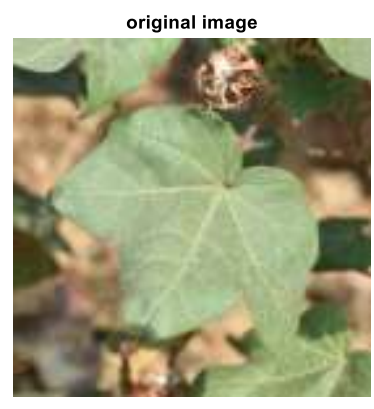

(a)

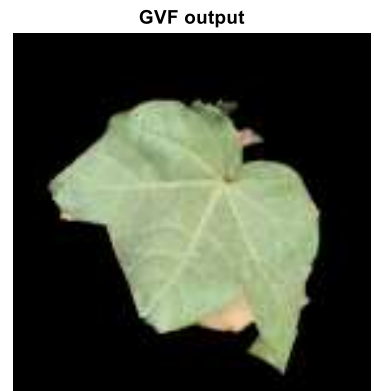

(c)

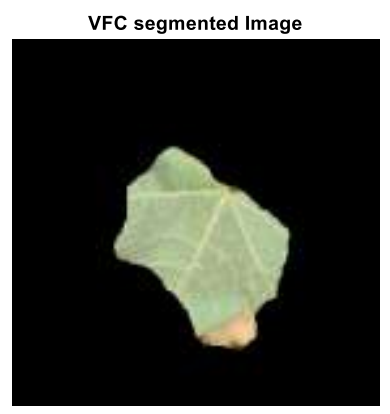

(d)

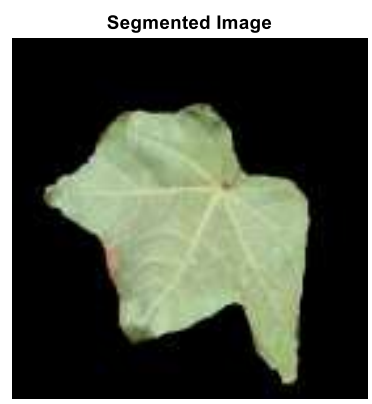

(b)
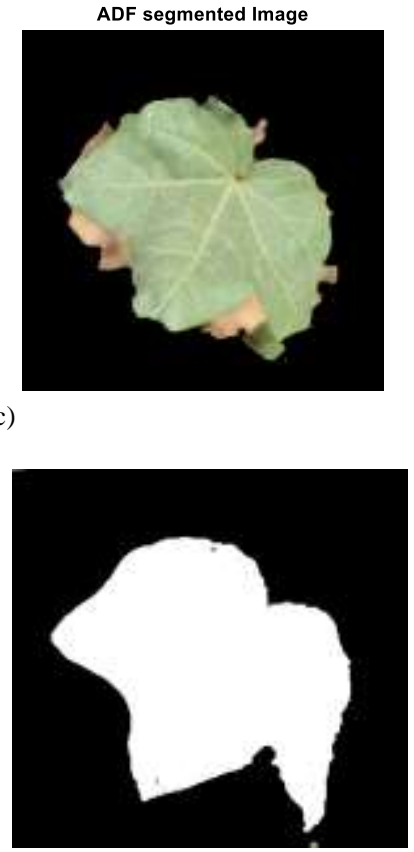

(e)
Fig. 4. (a) Input Image (b) MFACM Output (c) GVF Output (d) ADF Output (e) VFC Output (f) Ground Truth Image. 


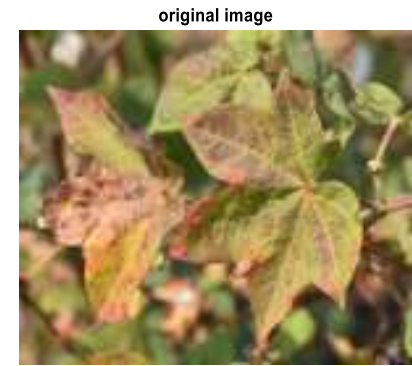

(a)

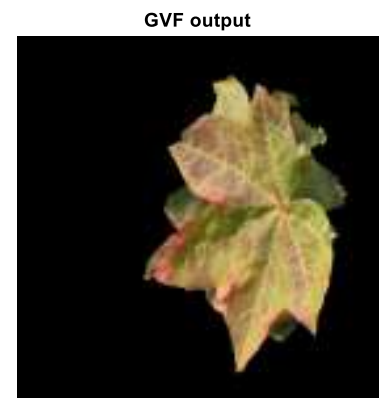

(c)

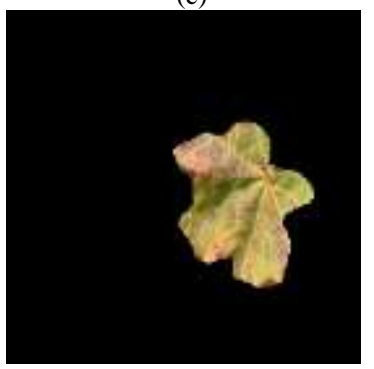

(e)

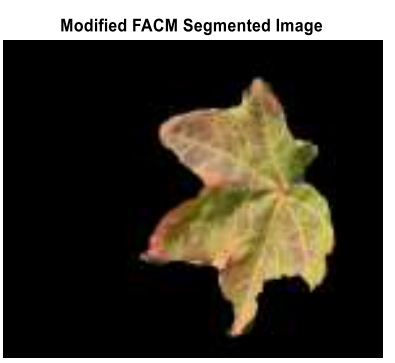

(b)

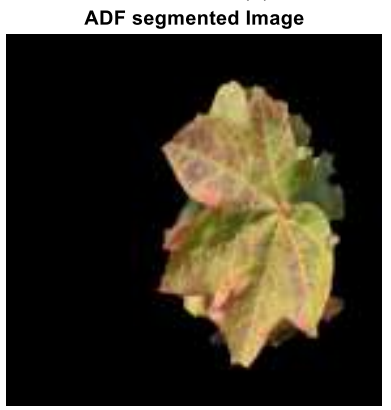

(d)

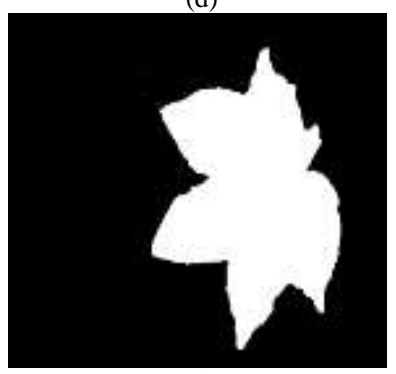

(f)
Fig. 5. (a) Input Image (b) Modified FACM Output (c) GVF Output (d) ADF Output (e) VFC Output (f) Ground Truth Image.

TABLE I. TIME SPENT FOR GVF, VFC, ADF AND MFACM

\begin{tabular}{|l|l|l|l|l|}
\hline Time spent & GVF & VFC & ADF & MFACM \\
\hline Iterations & $\mathbf{6 0}$ & $\mathbf{6 0}$ & $\mathbf{6 0}$ & $\mathbf{6 0}$ \\
\hline Leaf & 24.68 & 29.14 & 29.41 & 12.8 \\
\hline leaf1 & 23.73 & 40.67 & 32.54 & 11.06 \\
\hline leaf2 & 22.43 & 41.71 & 27.1 & 9.26 \\
\hline leaf3 & 23.63 & 37.01 & 30.91 & 11.59 \\
\hline leaf4 & 17.96 & 36.23 & 17.35 & 12.74 \\
\hline leaf5 & 21.68 & 29.32 & 22.92 & 11.54 \\
\hline
\end{tabular}

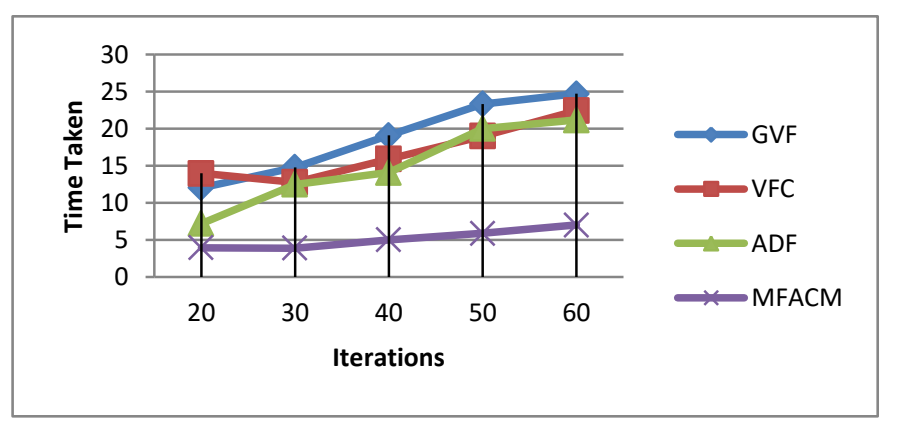

Fig. 6. Graph for Time Taken Versus Number of Iteration.
TABle II. False Positive, False Negative, True Positive, True Negative, ACCURACy, SpeCificity, SENSITIVITy, Precision VAlues

\begin{tabular}{|l|l|l|l|l|l|l|l|l|}
\hline & FP & FN & TP & TN & ACC & $\begin{array}{l}\text { SPE } \\
\text { CI }\end{array}$ & $\begin{array}{l}\text { SEN } \\
\text { SI }\end{array}$ & $\begin{array}{l}\text { Precisi } \\
\text { on }\end{array}$ \\
\hline GVF & $\begin{array}{l}202 \\
0\end{array}$ & 567 & $\begin{array}{l}221 \\
15\end{array}$ & $\begin{array}{l}408 \\
34\end{array}$ & $\begin{array}{l}0.96 \\
05\end{array}$ & $\begin{array}{l}0.952 \\
9\end{array}$ & 0.975 & 0.8864 \\
\hline ADF & 183 & $\begin{array}{l}111 \\
8\end{array}$ & $\begin{array}{l}215 \\
65\end{array}$ & $\begin{array}{l}410 \\
16\end{array}$ & $\begin{array}{l}0.95 \\
49\end{array}$ & $\begin{array}{l}0.957 \\
1\end{array}$ & $\begin{array}{l}0.950 \\
8\end{array}$ & 0.9215 \\
\hline VFC & 704 & $\begin{array}{l}114 \\
01\end{array}$ & $\begin{array}{l}113 \\
63\end{array}$ & $\begin{array}{l}420 \\
68\end{array}$ & $\begin{array}{l}0.81 \\
53\end{array}$ & $\begin{array}{l}0.983 \\
5\end{array}$ & $\begin{array}{l}0.499 \\
2\end{array}$ & 0.9417 \\
\hline MFAC & 682 & $\begin{array}{l}141 \\
4\end{array}$ & $\begin{array}{l}212 \\
68\end{array}$ & $\begin{array}{l}421 \\
72\end{array}$ & $\begin{array}{l}0.96 \\
8\end{array}$ & $\begin{array}{l}0.984 \\
1\end{array}$ & $\begin{array}{l}0.937 \\
7\end{array}$ & 0.9689 \\
\hline
\end{tabular}

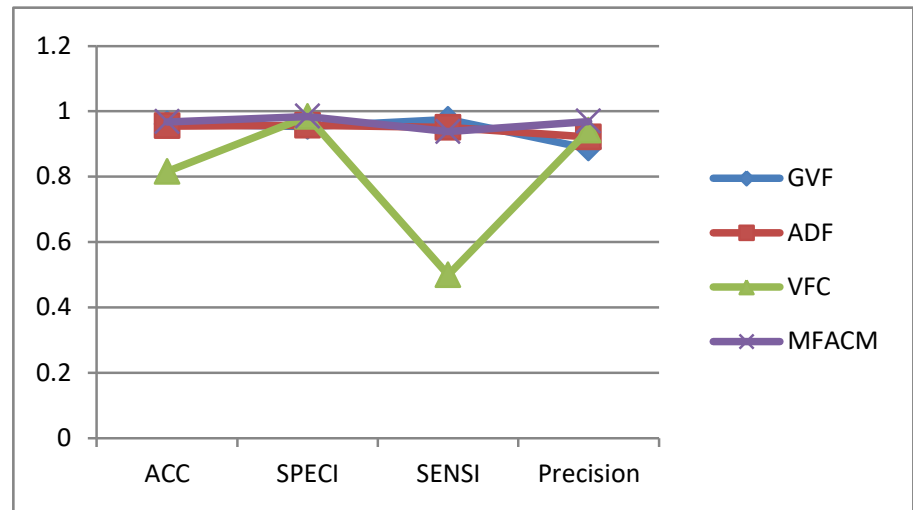

Fig. 7. Graph for Precision, Accuracy, Sensitivity, Specificity with Respect to MAFCM, ADF, VFC, GVF.

\section{CONCLUSION}

Leaf segmentation from complex background is performed using modified factorization based active contour for cotton leaf images. The segmented results are better than when compared to the parametric active contour methods. In this, active contour methods like GVF, VFC and ADF and level set method modified factorization based active contour method. From the results, we can observe that modified factorization based active contour method is good when compared to other methods in terms of time taken to perform segmentation. The precision, recall, sensitivity, etc. are calculated and the values are good when related to other methods. So, from this we can conclude that modified FACM is better than the other geometric and parametric active contour method.

\section{REFERENCES}

[1] Vijai Singh, A K Misra, "Detection of plant leaf diseases using image segmentation and soft computing techniques", Information processing in Agriculture, Volume 4, Issue 1, Pages 41-49,March 2017.

[2] Kiruba raji, K K Thyagharajan, "An analysis of segmentation techniques to identify herbal leaves from complex background", International Conference on Intelligent Computing Communication and Convergence (ICCC 2014), Odisha, India.

[3] J Praveen kumar, S. Domnic, " Image based leaf segmentation and counting in rosette plants", Information processing in Agriculture, Volume 6,Issue 2, Pages 233-246, June 2019.

[4] Manuel Grand-Brochier, Antoine Vacavant, Guillaume Cerutti, Camille Kurtz, Jonathan Weber, et al.,"Tree leaves extraction in natural images: Comparative study of pre-processing tools and segmentation Methods". IEEE Transactions on Image Processing, Institute of Electrical and Electronics Engineers,2015, 24 (5), pp.1549-1560. 
[5] Chenyang Xu and Jerry L. Prince, "Gradient vector flow: A external force for snakes", Proceedings of IEEE Computer Society Conference on Computer Vision and Pattern Recognition, 1997.

[6] Shivalika Sharma; Abhishek Gupta, "A review for the automatic methods of plant's leaf image segmentation", International journal of Intelligence and sustainable computing, Vol No 1, 2020.

[7] Hanno Scharr, Massimo Minervini, Andrew P French, Christian klukas, David M Kramer, xiaoming liu, Imanol Luengo, Jean Michel pape, Gerrit Polder, Danijela Vukadinovic , Xi yin, sotirious A Tsaftaris, "Leaf segmentation in plant phenotyping: a collation study",Machine vision applications,December 2015.

[8] Yuwei Wu, Yuanquan Wang, Yunde jia, "Adaptive diffusion flow active contours for image segmentation", computer vision and understanding, 2010.

[9] Bing Li, Scott T Action, “ Vector field convolution for Image segmentation using snakes", 2006 International Conference on Image Processing, IEEE.

[10] Xiaodong Tang, Manhua Liu, Hui Zhao, Wei Tao, "Leaf Extraction from Complicated Background", 2nd International Congress on Image and Signal Processing, IEEE, 2009.

[11] Mingqi Gao ,Hengxin Chen, Shenhai Zheng, Bin Fang, "A factorization based active contour model for texture segmentation", International conference on Image Processing, IEEE, 2016.

[12] Zhang Jian-hua, KONG Fan tao, WuJian zhai, HAN Shu-qing, ZHAI Zhi-fen ,"Automatic image segmentation method for cotton leaves with disease under natural environment", Journal of Integrative agriculture, 2018.

[13] Chunming Li, Chenyang Xu, Changfeng Gui, and Martin D ,"Level Set Evolution without Re-Initialization: A New Variational Formulation" Fox. IEEE CVPR, 2005.

[14] Jiange Yaun, Deliang Wang, Anil M Chariyadat, "Factorization based Texture Segmentation", IEEE Transaction on Image Processing", vol 24, Issue 11, 2015.

[15] Peng, Wu, Li Wenlin, and Song Wenlong. "Segmentation of Leaf images Based on Active Contour." International Journal of-u and-e Service, Science and Technology 8.6 (2015): 53-70.

[16] Srikham, Manassanan. "Active contours segmentation with edge based and local region based." Proceedings of the 21 st International Conference on Pattern Recognition (ICPR2012). IEEE, 2012.
[17] Zhang, Shanwen, et al. "Plant diseased leaf segmentation and recognition by fusion of superpixel, K-means and PHOG." Optik 157 (2018): 866-872.

[18] V. Caselles, F. Catte, T. Coll, and F. Dibos, "A geometric model for active contours in image processing",Numer.Math., vol. 66, pp. 1-31, 1993.

[19] Cerutti G., Tougne L., Vacavant A., Coquin D., "A Parametric Active Polygon for Leaf Segmentation and Shape Estimation, Advances in Visual Computing. ISVC 2011.

[20] J. A. Sethian,Level Set Methods and Fast Marching Methods: Evolving Interfaces inComputational Geometry, Fluid Mechanics, Computer Vision, and Material Science.Cambridge, UK: Cambridge University Press, 2nd ed., 1999.

[21] I. Cohen, L. D. Cohen, and N. Ayache, "Using deformable surfaces to segment 3-D images and infer differential structures,"CVGIP: Imag. Under., vol. 56, no. 2,pp. 242-263, 1992.

[22] Jonas De Vylder, Daniel Ochoa, Wilfried Philips, Laury Chaerle, and Dominique Van Der Straeten, "Leaf segmentation and tracking using probabilistic parametric active contour", vol 6930, 2011.

[23] Xu, Chenyang, and Jerry L. Prince. "Gradient vector flow." Computer Vision: A Reference Guide (2020): 1-8.

[24] Eltanboly, Ahmed, et al. "Level sets-based image segmentation approach using statistical shape priors." Applied Mathematics and Computation 340 (2019): 164-179.

[25] Kumar, J. Praveen, and S. Domnic. "Image based leaf segmentation and counting in rosette plants." Information Processing In Agriculture 6.2 (2019): 233-246.

[26] Wang, Ping, et al. "An maize leaf segmentation algorithm based on image repairing technology." Computers and Electronics in Agriculture 172 (2020): 105349.

[27] Zhang, Shanwen, Zhuhong You, and Xiaowei Wu. "Plant disease leaf image segmentation based on superpixel clustering and EM algorithm." Neural Computing and Applications 31.2 (2019): 1225-1232.

[28] Tian, Kai, et al. "Segmentation of tomato leaf images based on adaptive clustering number of K-means algorithm." Computers and Electronics in Agriculture 165 (2019): 104962.

[29] S. Osher and J. A. Sethian, "Fronts propagating with curvaturedependent speed: algorithms based on Hamilton-Jacobi formulations", J.Computational physics, vol 79,pp. 12-49, 1988. 